\title{
Using Enterprise Models to Explain and Discuss Autopoiesis and Homeostasis in Socio-technical Systems
}

\author{
Ilia Bider ${ }^{{ }^{*}}$, Gil Regev ${ }^{2}$, and Erik Perjons ${ }^{1}$ \\ ${ }^{1}$ Department of Computer and Systems Sciences (DSV), Stockholm University, \\ 16455 Kista, Sweden \\ ${ }^{2}$ Ecole Polytechnique Fédérale de Lausanne (EPFL), School of Computer and \\ Communication Sciences, CH-1015 Lausanne, Switzerland \\ ilia@dsv.su.se, gil.regev@epfl.ch, perjons@dsv.su.se
}

\begin{abstract}
The article links two seemingly different fundamental theoretical concepts of autopoiesis and homeostasis and tries to apply them to the realm of socio-technical systems with the use of the Fractal Enterprise Model (FEM). Autopoiesis is the property of a system that constantly reproduces itself. Homeostasis describes a way a complex system constantly maintains its identity while adapting to changes in its internal and external environment. To be able to use FEM for this task, the original version of FEM has been extended by adding special elements for representing the system's context - part of the environment to which the system is structurally coupled. The approach taken in this article differs from other works in the same field in having the focus on the "body" (concrete elements being reproduced) of the socio-technical system, as well as on identifying concrete processes that reproduce the system, and demonstrating concrete ways of how a specific system adapts or can adapt to the perturbations in the environment (i.e. internal and external disturbances that affect the system).

Keywords: Socio-technical, Autopoiesis, Homeostasis, Structural Coupling, Fractal Enterprise Model.
\end{abstract}

\section{Motivation}

The term autopoiesis comes from biological cybernetics, first introduced in [1], [2] to identify the particulars of living systems that differentiate them from other types of systems. The term means that a living system constantly reproduces itself. More exactly, an autopoietic living system/organism, according to the original authors who coined the term, consists of a network of molecular processes that constantly reproduce the components of the system.

Though the term autopoiesis was introduced for describing biological systems, it was soon reinterpreted for other system types; the first of these were sociological systems. The most

\footnotetext{
* Corresponding author

(C) 2020 Ilia Bider et al. This is an open access article licensed under the Creative Commons Attribution License (http://creativecommons.org/licenses/by/4.0).
}

Reference: I. Bider, G. Regev, and E. Perjons, "Using Enterprise Models to Explain and Discuss Autopoiesis and Homeostasis in Socio-technical Systems," Complex Systems Informatics and Modeling Quarterly, CSIMQ, no. 22, pp. 21-38, 2020. Available: https://doi.org/10.7250/csimq.2020-22.02

Additional information. Author ORCID iD: I. Bider - https://orcid.org/0000-0002-3490-6092, E. Perjons https://orcid.org/0000-0001-9044-5836. PII S225599222000127X. Received: 13 December 2019. Accepted: 12 February 2020. Available online: 30 April 2020. 
known application of the concept of autopoiesis to the realm of the social world is the one introduced by N. Luhmann [3]. Luhmann identifies two types of autopoietic systems, a system of communication, and a system of decisions (an organization). The first one always produces new communication acts based on already existing ones, the second constantly produces new decisions based on already made ones. Luhmann was not the only one who applied the concept of autopoiesis to the social realm. The literature on this topic is vast; it includes books, e.g. [4] and articles; and it encompasses different ideas of what is being reproduced by a system. One of the most used ideas of what is to be reproduced, besides what Luhmann has proposed, is knowledge, see, for instance, [5].

Using the concept of autopoiesis outside biology is still a controversial issue. The opinions on this issue range from disagreement from the original authors of the original concept, to considering any autopoietic system as being a social system. The latter is promoted by Milan Zeleny and his group, see, for instance, [6] where they present examples of social systems showing that they all satisfy the 6 point test suggested in [1]. In this respect, it is interesting to read [7] which includes post-reviews from the major personalities related to the concept of autopoiesis, including Humberto R. Maturana and Milan Zeleny.

Though the original concept of autopoiesis in biological systems was focused on the reproduction processes, what is being reproduced is also considered important. This is true even for biology itself, see, for instance, [8], which argues that considering an autopoietic system just as a network of processes is not enough; the body of an organism should also be taken into consideration.

The question that we want to raise in this article is whether it is possible to apply the concept of autopoiesis to socio-technical systems. As a socio-technical system is considered as consisting of two components social and technical, applying the concept of autopoiesis to such systems implies reproducing both components. Moreover, if we consider that each component can be split in two parts according to the classical Socio-Technical System (STS) matrix [9], applying the concept of autopoiesis means reproducing, people, structure, tasks and technology. In summary, when applying the concept of autopoiesis to a socio-technical system, such as an enterprise, we need to answer two questions:

1. What is reproduced (the concrete elements of the socio-technical system, i.e. the "body" of the system)?

2. How is it done (network of processes involved in the reproduction)?

Maturana and Varela made the link between autopoiesis and homeostasis by saying, early in [2], that "Autopoietic machines are homeostatic machines". We, therefore, consider the third question:

3. How the autopoietic activities help with homeostasis, i.e. adapting the system to the changes inside and outside the system while maintaining its identity?

This article has an explanatory nature, the goal being to give a somewhat new view on organizational systems. While answering the above questions, we do not focus on how the answers could help in solving practical problems, though we do give some hints about it. The practicality of the results depends on the background and experience of the reader, as well as on the task at hand. Investigating all possible usage of the suggested view is outside the scope of this article, but the task is considered for the future.

In this article, following [10], we use the POSWID principle of looking at an organization. The POSWID principle was coined by Stafford Beer in connection to his Viable System Model [11], and the abbreviation stays for "the Purpose Of a System Is What It Does". In practical terms, we do not consider a stated purpose of an organization, e.g. its mission and vision, and how it is being implemented, but concentrate on looking at the organization's operational activities. To complete such an investigation, we use an enterprise model that represents the operational activities in a suitable form. More specifically, in this investigation, we use a special technique for enterprise modeling called Fractal Enterprise Model (FEM) [12]. FEM has a form 
of a directed graph with two types of nodes Processes and Assets, where the arrows (edges) from assets to processes show which assets are utilized by which processes and arrows from processes to assets show which processes help to have specific assets in "healthy" and working order. The arrows are labeled with meta-tags that show in what way a given asset is utilized, e.g. as workforce, reputation, infrastructure, etc.; or in what way a given process helps to have the given assets "in order", i.e. acquire new elements to fill an asset, maintain existing elements or retire elements that no longer can be used in the process.

A FEM is built recursively by using a so-called unfolding procedure and two types of archetypes: process-assets archetypes that show, which kind of assets might be needed for running a process, and an asset-processes archetype that shows which processes are needed to maintain an asset in order. Unfolding starts with a primary process - a process that delivers value to a customer/beneficiary - by applying process-assets archetypes and alternating them with the asset-processes archetype.

The presence of processes that manage assets (acquire, maintain and retire) makes FEM interesting when considering autopoiesis and homeostasis in socio-technical systems. These processes are the ones that constitute the net of processes responsible for autopoiesis. They, also, are the ones that can inform the system of changes in its internal or external environment.

The rest of the article is structured in the following manner. In Section 2, we present our knowledge base, which includes Zeleny's approach to describing processes in autopoietic systems [6], the notion of homeostasis and FEM. In Section 3, we use FEM to answer the first two questions posed above. Section 4 is devoted to answering the third question. Section 5 discusses how our approach differs from the majority of works devoted to the same theme. Section 6 contains concluding remarks and plans for the future.

This article is an extended version of the paper [13] presented at STPIS 2019. Based on the presentation and the following discussion, FEM was extended in order to better reflect the nature of an organization as an autopoietic and homeostatic system.

\section{Knowledge Base}

\subsection{General Processes in Autopoietic Systems}

According to Zeleny [6], there are three general types of processes in an autopoietic system: (1) Degradation, (2) Production, and (3) Bonding, see Figure 1. Production is a process of creating new components. Bonding is a process of introducing new components into the system structure. Degradation is a natural process of components aging and falling out of the system structure, which requires production of new components to be bound into the structure. The specific meaning of these generic processes depends on the system in question. In a post-review by Zeleny [7] there are several examples of instantiation of the generic process.

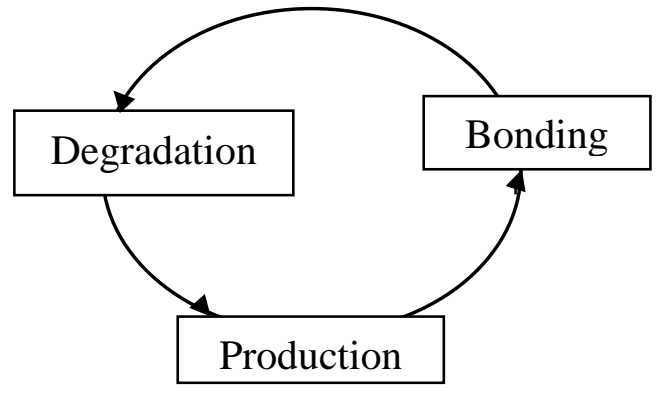

Figure 1. Generic processes in an autopoietic system, adapted from [6] 


\subsection{Homeostasis}

The concept of homeostasis predates autopoiesis by about 40 years. It was defined by Cannon in the 1920s [14] based on research done during the 19th century by Claude Bernard. Just like Maturana and Varela, Cannon (and other physiologists) attempted to explain the mystery of life.

Cannon stipulated that what distinguishes the living organism is its ability to maintain constancy despite it being subjected to internal and external perturbations. This constancy, Cannon claimed, is the source of freedom of action [15], [16]. The better the living organism maintains its constancy the more it can take control of its environment. Mammals, for instance, have better mechanisms than reptiles for maintaining the constancy of their body temperature and are therefore better able to function irrespective of the ambient temperature.

Autopoiesis can be seen as a special case of homeostasis because constantly reproducing itself is one consequence of the maintenance of global constancy. Homeostasis is also more general because it applies to any open system that is [14]: "compounded of unstable material and subjected continually to disturbing conditions", i.e. systems that are subject to the second law of thermodynamics, the increase of entropy (disorder) in any closed portion of the universe [16].

Autopoiesis, on the contrary, is principally applied to the description of living systems, which it defines as closed systems.

Cannon enounced 6 propositions that define a homeostatic system [15], [16], that run as follows:

In an open system, such as our bodies represent, compounded of unstable material and subjected continually to disturbing conditions, constancy is in itself evidence that agencies are acting or ready to act, to maintain this constancy.

If a state remains steady it does so because any tendency towards change is automatically met by increased effectiveness of the factor or factors which resist the change.

Any factor which operates to maintain a steady state by action in one direction does not also act at the same point in the opposite direction.

Homeostatic agents, antagonistic in one region of the body, may be cooperative in another region.

The regulating system which determines a homeostatic state may comprise a number of cooperating factors brought into action at the same time or successively.

When a factor is known which can shift a homeostatic state in one direction it is reasonable to look for automatic control of that factor, or for a factor or factors having an opposing effect.

The first proposition is most important for our present discussion, as according to it, homeostasis explains both the process of degradation (unstable material and disturbing conditions) and the need for process of replacement, production and bonding, (maintaining constancy). It also shows that both the internal environment and the external environment are sources of change, mostly unwelcome change, with which the system attempts to deal in order to maintain its constancy.

Cannon envisioned that homeostasis could be applicable by analogy to the study of social systems [15], [16] and even socio-technical systems [15]. He, for instance, foresaw that technical innovations can remove the jobs of scores of people, leading to the need to restore (maintain the constancy of their life conditions) by providing unemployment benefits and retraining [15]. 


\subsection{Fractal Enterprise Model}

\subsubsection{YAMCC - Just Yet Another Management Consulting Company}

Explaining the structure of Fractal Enterprise Model (FEM), as well as discussing autopoiesis and homeostasis of organizational systems, we use an example of an idealized management consulting company called YAMCC (Yet Another Management Consulting Company). YAMCC itself is a fictive company, but it represents a prototypical image of a management consulting company built based on the features we encountered when building a FEM model for a real management consulting company. YAMCC provides help to its customers in solving various management problems. As in any other typical consulting company, the service is completed by sending management consultants to the customer sites to conduct investigations and come up with solutions. The consultants use methods in conducting investigations and designing solution, as well as tools that support the methods. Some tools could be implemented as software packages. Methods and tools could be developed inside the company, or adopted/bought from the third parties.

An idealized image of a management consulting company and presenting the minimum of details were chosen in order not to complicate the discussion with particulars of a specific company. The latter may distract the reader's attention from the main topic of this article. Readers who are interested in viewing examples from specific organizations are referred to other works devoted to FEM technique, e.g. [12], [17], [18].

\subsubsection{Original Fractal Enterprise Model}

The original version of FEM from [12] includes three types of elements: business processes (more exactly, business process types), assets, and relationships between them, see Figure 2, in which a fragment of the model related to YAMCC is presented. Graphically, a process is represented by an oval, an asset is represented by a rectangle (box), while a relationship between a process and an asset is represented by an arrow. We differentiate two types of relationships in the fractal model. One type represents a relationship of a process "using" an asset; in this case, the arrow points from the asset to the process and has a solid line. The other type represents a relationship of a process changing the asset; in this case, the arrow points from the process to the asset and has a dashed line. These two types of relationships allow tying up processes and assets in a directed graph.

In FEM, a label inside an oval names the given process, and a label inside a rectangle, names the given asset. Arrows are also labeled to show the type of relationships between the processes and assets. A label on an arrow pointing from an asset to a process identifies the role the given asset plays in the process, for instance, workforce and infrastructure. A label on an arrow pointing from a process to an asset identifies the way in which the process affects (i.e. changes) the asset. In FEM, an asset is considered as a pool of entities capable of playing a given role in a given process. Labels leading into assets from supporting processes reflect the way the pool is affected, for instance, the label Acquire identifies that the process can/should increase the pool size.

Note that the same asset can be used in several different processes playing the same or different roles in them, which is reflected by labels on the corresponding arrows. It is also possible that the same asset can be used for more than one role in the same process. In this case there can be more than one arrow between the asset and the process, but with different labels. Similarly, the same process could affect different assets, each in the same or in different ways, which is represented by the corresponding labels on the arrows. Moreover, it is possible that the same process affects the same asset in different ways, which is represented by having two or more arrows from the process to the asset, each with its own label. When there are too many arrows leading to the same process or asset, several copies can be created for this process or asset in the diagram. In this case, the shapes for copies have a bleaker color than the original, see asset "Reputation of a good problem solver" in Figure 2. 


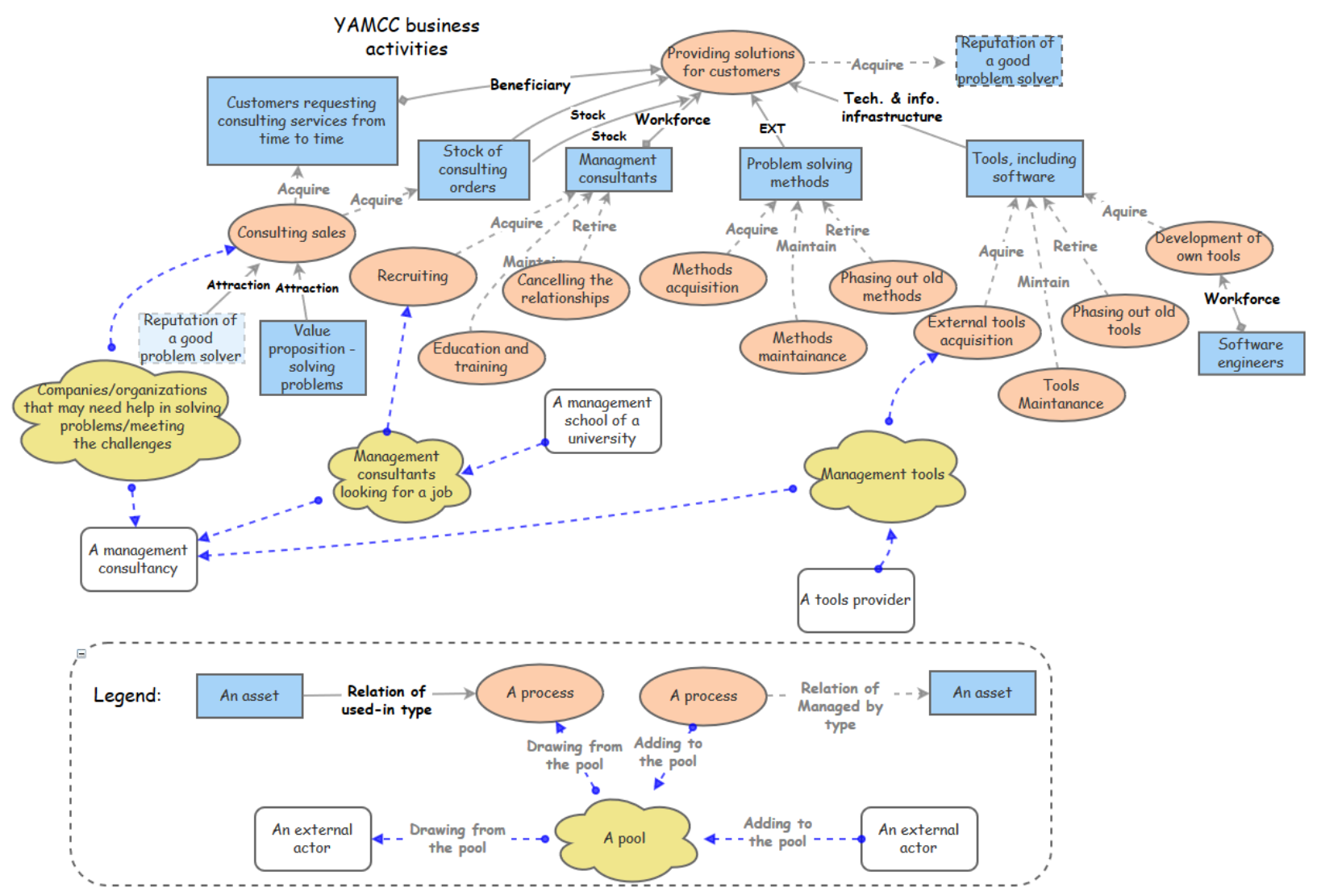

Figure 2. A fragment of a FEM for YAMCC - a management consulting company

In FEM, different styles can be used for shapes to group together different kinds of processes, assets, and/or relationships between them. Such styles can include dashed or double lines, or lines of different thickness, or colored lines and/or shapes. For instance, a diamond start of an arrow from an asset to a process means that the asset is a stakeholder of the process (see the arrows "Workforce" in Figure 2).

Labels inside ovals (which represent processes) and inside rectangles (which represent assets) are not standardized. They can be set according to the terminology accepted in the given domain, or be specific for a given organization. Labels on arrows (which represent the relationships between processes and assets) can be standardized. This is done by using a relatively abstract set of relationships, such as, Workforce or Acquire, which are clarified by the domain- and contextspecific labels inside ovals and rectangles. Standardization improves the understandability of the models.

While there are several types of relationships that show how an asset is used in a process (see example in Figure 1), there are only three types of relationships that show how an asset is managed by a process - Acquire, Maintain, and Retire.

A FEM shows both the structure of the enterprise, and its content. The structure is revealed through relationships between assets and processes, while the body is revealed if we look inside each asset, i.e. on concrete elements that are included in it.

To make the work of building a fractal model more systematic, FEM uses archetypes (or patterns) for fragments from which a particular model can be built. An archetype is a template defined as a fragment of a model where labels inside ovals (processes) and rectangles (assets) are omitted, but arrows are labelled. Instantiating an archetype means putting the fragment inside the model and labelling ovals and rectangles; it is also possible to add elements absent in the archetype, or omit some elements that are present in the archetype.

FEM has two types of archetypes: a process-assets archetype and an asset-processes archetype. The process-assets archetype represents the kinds of assets that can be used in a given category 
of processes. The asset-processes archetype shows the kinds of processes that are aimed at changing the given category of assets.

Note that in FEM each process node is connected to assets representing different sides of a sociotechnical system, e.g. people (workforce) and technology (technical and informational infrastructure). However, there is no explicit mentioning of these assets being aligned with each other. Implicitly such alignment is necessary for a process being able to function. Moreover, changes in any of the assets connected to a particular process node, e.g. people or technology, require readjustment of other assets connected to the node. This issue is covered in more details in [19].

\subsubsection{Extended Fractal Enterprise Model}

When testing FEM as a tool for explaining autopoietic and homeostatic nature of organizations, we have discovered the needs to extend FEM. Two new concepts were introduced for this end:

- External pool, which is represented by a cloud shape, see Figure 2. An external pool is a set of things or agents of a certain type. As an example, in Figure 2, there are three such pools: (1) pool of organizations that need help from a consulting company, (2) pool of management consultants looking for a job, (3) pool of management tools that can be employed by management consultants. The label inside the external pool describes its content.

- External actor, which is represented by a rectangle with rounded corners. An external actor is an agent, like a company or a person, acting outside the boundary of the organization. The label inside the external actor describes its nature. If a label starts with indefinite article "a" or "any", the box represents a set of agents of the given type, see Figure 2, which have three external actors of this kind, that is, A management consultancy, A management school of a university, and A tool provider.

External pools and external actors may be related to each other and to other elements of the FEM diagram. Such a relation is shown by a dashed arrow that has a round dot start. More exactly:

- A business process may be connected to an external pool with an arrow directed from the pool to the process. In this case, the process needs to be an acquire process to one or more assets. The arrow shows that the process uses the external pool to create new elements in the asset for which this process serves as an acquire process, see, for instance, three incoming arrows from external pools into processes Consulting sales, Recruiting, and External tool acquisition in Figure 2.

- An external actor may be connected to an external pool with an arrow directed from the pool to the external actor. In this case the arrow shows that the external actor uses the external pool as the basis for one of its own acquire processes, see, for instance, three incoming arrows into external pool A management consultancy in Figure 2.

- A business process may be connected to an external pool with an arrow directed from the process to the pool. In this case the arrow shows that the process provides entities to the external pool. There are no examples of such relations in Figure 2; an example of this type will be introduced later.

- An external actor may be connected to an external pool with an arrow directed from the actor to the pool. In this case the arrow shows that one of the actor's processes provides entities to the external pool, see, for instance, the arrow from external actor $A$ tool provider to external pool Management tools in Figure 2.

External pools and actors represent the context in which an organization operates. External pools can be roughly associated with markets, e.g. a labor market, management tools market, etc. External actors represent other organizations that are connected to the external pools. Depending on the nature of the external pool an external actor connected to it can be a competitor, provider or collaborator. 


\section{What Components are Reproduced and How?}

The basic idea of FEM is that any process (type) needs assets in order to be able to run its instances with a required regularity. These assets age/become depleted with time and need renovation/service or substitution with new ones, which is in total agreement with the first of Cannon's six propositions of homeostasis [14] of the body consisting of unstable components and the needs for action to maintain consistency. The substitution requires retiring old/depleted assets along with the introduction of the new ones. The assets management processes are attached to each asset with a dashed arrow and the labels Acquire, Maintain or Require. Without the management processes in place, the asset will be depleted and the process(es) that uses this asset will no longer be able to run new instances.

Note that a primary process in FEM also serves as an asset management process. For instance, the root process in Figure 2, Providing solutions for customers, can be considered as a process of acquiring monetary funds and reputation of a reliable management consultant company. The asset "monetary funds" is an asset that is needed for all processes (not represented in Figure 2), while the "asset" "reputation" is needed for sales and marketing - a customer acquiring process (see Consulting sales and the Attraction arrows in Figure 2).

Based on the discussion above, we can conclude that a FEM model of an organization describes both the "body" of the organization - its assets, and the processes that are responsible for reproducing the body. Note that the concept of a body derived from FEM includes more than a traditional drawing of an organizational boundary, as it may include assets that, strictly speaking, are outside its full control, such as customers or partners (e.g. suppliers).

The generic reproduction idea in FEM is similar to the one suggested by Zeleny in Figure 1, but it is slightly different. We do not syntactically differentiate production and bonding. The Acquire arrow is used for both purposes. On a high-level of details, an acquire process may denote both getting new entities for an asset pool and introducing them in the structure of the functioning organization. As an example, in Figure 2, Recruiting serves to produce new management consultants as employees and introduces them into organizational practice. In a more detailed diagram, see Figure 3, this process can be split into two: one to obtain new employees to the company and another - to introduce them into practice. The first process can be roughly considered as production, and the second - as bonding.

The other difference from Zeleny's generic framework is the addition of explicit maintenance processes, a very important type of process in any system. Broken expensive equipment is being repaired rather than discarded as soon as it malfunctions. Moreover, the equipment is being serviced at certain points to ensure it being in working order. Bugs in software are fixed rather than developing new software. People suffering from burn-out are rehabilitated, rather than being substituted by new hires. As maintenance, we also consider processes of alignment within a socio-technical system when one of its components has been changed. For instance, substituting an old software system may require retraining the people who worked with the old one, as well as adjusting the documentation related to the processes in which the system is employed.

From a systems perspective, a process node of FEM, with supporting assets, represents a work system responsible for initiating and finishing process instances of the given type. We call this system a Business Process Work System or BPWS for short. BPWS can be regarded as a sociotechnical system as it includes people, methods, e.g. manuals that prescribes the process flow, technology, and structure - distribution of responsibilities between the members of the team responsible for the process. Note that the term work system was introduced by S. Alter, see, for instance, [20], and BPWS can be considered as a particular class of a work system. 


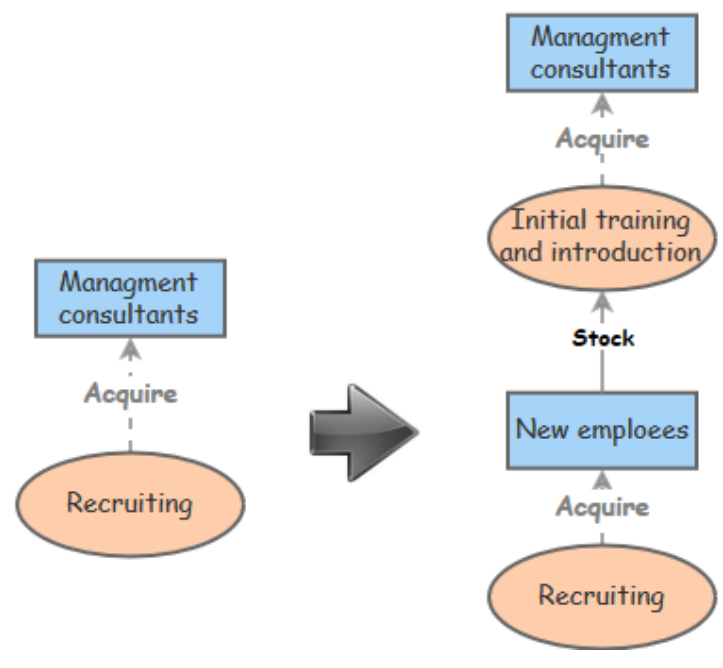

Figure 3. More detailed FEM fragment for acquiring new workforce agents

A process instance is initiated based on a combination of external and internal conditions. An example of an internal condition for an acquire process is a resource depletion, real or expected. Let us illustrate how internal conditions work using the example in Figure 2. When some of the management consultants suddenly leave, the internal pool of consultants (the asset that plays the role of workforce) becomes somewhat depleted, which gives a signal to starting an instance of the Recruiting process. Another internal signal to this end is when some consultant(s) is nearing the retirement age and will need to be substituted. Recruiting a new consultant gives a signal to the Initial introduction and training from Figure 3 to start an instance of this process.

Note that a Business Process Instance (BPI) can be also considered as a system that is created to handle a specific situation defined by a condition for creating an instance. This system can be considered as a respondent system in terms of [21] that is created to handle a specific situation and that is disbanded when the situation is resolved. When creating a BPI, BPWS gives it some of its assets to be engaged in the BPI. It also follows the work of BPI, and, if needed, can give more assets. After the BPI is completed, all assets are returned back to the BPWS. Note that assets may not be given to a BPI exclusively, but may be shared with other BPIs. More on systems theoretical underpinning of FEM can be found in [12], [19].

\section{Linking Autopoiesis to Homeostasis}

In this article, we consider homeostasis as an ability of a socio-technical system to (a) adapt itself to changes in the internal and external environment, while (b) maintaining its identity. These two sides of homeostatic behavior are considered in the following sub-sections. Note that, the reproduction of the body that can be considered as part of the homeostatic activities is not considered in this section, as it was already discussed in Section 3.

\subsection{How the Adaptation Happens}

While Section 3 explains how autopoiesis - body reproduction - works in a socio-technical system, it does not explicitly refer to the need for adaptation. The procedure of reproducing the system's "body" seems to work in a way that the body, essentially, does not change. The depleted assets are being replenished by the same kind of entities, i.e. the same kind of customers, employees, technology, etc. This is not true in reality, as the replenishing the assets is often done by obtaining components from the outer world and bonding (the term used by Zeleny [6]) them into the system. This dependence on the external world is represented by external pools in the FEM diagram, see Figure 2. 
An external pool changes in time, thus new components taken by an acquire process instance in order to replenish an asset may have different characteristics than in previous instances. This difference can affect the system in various way. For instance, to acquire new customers (beneficiaries in terms of FEM), there might be a need to change the value proposition, as the customers' needs in the market have changed. This in turn, will require changing the product and/or service assortment, which will initiate changes along the design and delivery branch of the FEM tree. For instance, for the consulting company in Figure 2, a change in the value proposition may require changing methods and tools. Note that the needs to adjust the offering may also be discovered by the customer maintenance process, i.e. the offering (value proposition) might need to be changed to retain the existing customers (this process is not depicted in Figure 2).

The same process for adapting to changes can be invoked by an acquire process for any other asset that requires input from the environment. For instance, when new equipment is purchased (the infrastructure type of assets), it may be more advanced than the previous one. This can lead to the need to retrain the staff (a maintenance process), and/or lead to the need to decrease the number of people employed (activates the retiring processes).

Summarizing the discussion above, any of the acquiring processes that need to incorporate components from the environment into the system, i.e. the one that is connected to an external pool, may initiate a chain of changes that leads to the system adapting to the induced change. This is done in order to maintain the system's identity by moving from a state that become unstable to a different but stable state. The components that are obtained from the outer world may concern any side of the socio-technical system: people (hiring), technology (purchasing), methods (executable templates) and structures (organizational infrastructure, not shown in Figure 2). Moreover, the change initiated in one place may affect other parts of the system. To propagate the changes BPWSs needs to communicate with each other, especially if they are connected to each other in the FEM graph. This topic is exploited in more details in [19].

\subsection{What Remains Constant}

In the previous section, we used FEM to explain how adaptation happens while the system reproduces itself by acquiring components from the outer world. This, however, covers only one side of the concept of homeostasis - adaptation. The other side is the system maintaining identity while adapting itself to the changes in the environment. This, in turn, leads to the question of what constitutes identity of a socio-technical system. There is a vast literature on the concept of organizational identity starting with the seminal work [22] from 1985, revisited in [23] by one of its authors in 2006. However, in this work, we prefer to stay with the FEM model when discussing organizational identity.

Whether the identity is maintained or changed depends on the observer, which can be an external or internal agent. Each observer can be considered as a system on its own, and it sees the world using its own lenses. This fact is well-known from biology, e.g. a frog sees mainly small objects moving with a speed and may miss a bigger object moving slowly [24]. The same is true for the observers of an enterprise or any other organization.

Consider, for instance, that our observer 1 belongs to pool Companies/organizations that may need help in solving problems/meeting the challenges in Figure 2, i.e. a pool of potential customers. For this observer, YAMCC remains the same as long as its value proposition is the same "providing (management) solutions" and it has a good reputation. Changes in the methods, tools, and management consultants would not affect much the identity of YAMCC for this observer. The same is true if our observer 2 is an external agent of the type A management consultancy from Figure 2. This observer represents a competitor to YAMCC, and it sees YAMCC as a competitor (though it does not prohibit them to collaborate). Changes in the methods and tools will not change the identity of our company for observer 2. Let us consider 
observer 3 as a member of the pool Management consultants looking for a job. The identity of JYACCM will be preserved as long as it is preserved for observers 1 and 2.

Consider now that we have observer 4 that is an agent of the type A tools provider. As long as YAMCC has a process External tools acquisition that draws new tools from the Management tools pool, the identity of YAMCC will be the same. More exactly, observer 4 considers YAMCC as a potential customer (unless YAMCC is already a customer). Suppose that YAMCC decided not to use any tools from the market and rely only on tools developed in the house. This means that process External tools acquisition disappears. This will not affect the identity of YAMCC for observer 1, 2 and 3, but it will affect its identity for observer 4 . In this case YAMCC will not be connected to pool Management tools, and thus ceases to be a potential customer. It will disappear from the "radar" of observer 4, which will not see YAMCC in the same way as a frog does not see bigger slowly moving objects.

Consider now that a YAMCC's developed tool becomes much appreciated by their customers, which start asking YAMCC permission to use the tool themselves. Based on this experience, YAMCC decided to start a new primary business process of licensing their management tool. A FEM fragment related to this new business activity is presented in Figure 4. In this case, YAMCC will reappear on the "radar" of our Observer 4 but this time as a competitor, as it will add a new product to the Management tools market. Observer 2 might also discover the change if it belongs to Tool seekers pool in Figure 4.

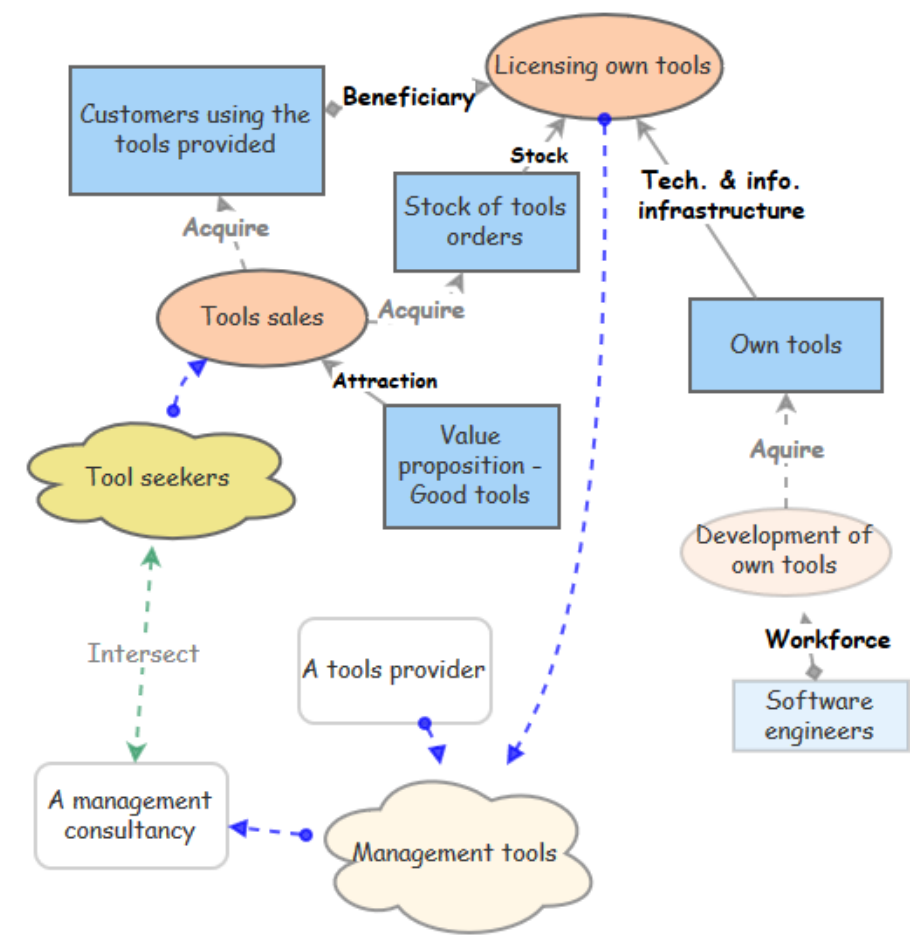

Figure 4. A new primary business process at YAMCC

Let us continue our discussion by supposing that Licensing own tools activity was very successful and the YAMCC management decided to concentrate on this business and drop management consulting altogether. In this case observers 1,2 and 3 will see the change. For observer 1, the YAMCC ceases to exist as a management consultancy, but might remain in the view as a tool vendor. For observer 2, YAMCC ceases to exist as a competitor, but might remain in the view as a tool vendor. For observer 3, the company ceases to exist as a potential workplace. For observer 4 the identity of the company stays the same, as for this observer, the business activity that has been dropped has no relevance.

Note also, that besides the external observers, there are internal ones, customers (who are a part of the extended body), employees and partners who can also detect or not detect changes in the identity. For instance, for management consultants working for the company, the last change 
can be considered as an identity change, as they need to leave the company, or change the position in it. For instance, they can become members of a support team that helps the new customers to learn and use the tools sold by YAMCC.

The concept of structural coupling [25], closely related to autopoiesis, gives an idea about which external observers will be affected by an identity change and which will not. The idea of structural coupling is relatively simple. There are elements of the environment that are more tightly connected to the given system (organization) than other parts of the system's environment. The system focuses on reacting to changes in these elements or trying to change them, while more or less ignoring other elements (systems) in the environment. Applying the idea of structural coupling to defining organizational identity was suggested in [10]. It amounts to defining the maintenance of identity as maintaining structural coupling with the important elements of the environment. Our experience of using this idea [26] showed that it was quite useful in practice, which is one of the reasons we use it in this article.

As we have already discussed, several acquiring processes in an organization are connected to external pools from which they take elements to fill depleted assets. We can consider that the organization is structurally coupled to, at least, some of these pools, and through them to agents that operate on the pools, deriving components from it (potential competitors) or adding components to it (providers). The main goal of external adaptation can be considered as maintaining the structural coupling to these external pools and agents. If the structural coupling remains, we can consider that the identity has not changed despite changes implemented in the organization. However, as we have seen from the examples, the company may change its structural couplings from time to time by making coupling to other external pools and agents, and later dropping the previously existing structural coupling.

To conclude this section, we can generalize the concept of social-technical (organizational system) adaptation in the following way:

1. An organizational change that does not change structural couplings to the external environment can be considered as adaptation not changing the identity for any structurally coupled external observer. However, some internal observers and external observers that are not part of structurally coupled external pools and agents can consider the change as an identity change.

2. Organizational change that affects structural couplings by adding new couplings and/or removing others can be considered as an adaptation if the majority of the existing structural couplings remain intact. This change does not change the overall identity of the system, though some external observers that belong to the structurally coupled pools and agents consider that the system has changed its identity.

According to the above, a system maintains its identity by preserving the majority of its structural couplings while adapting to internal and external changes. This may lead to a system having a completely different set of structural couplings after some time. Still we can consider it as preserving its identity at each step of its evolution.

\subsection{How Cannon's Propositions are Realized in a Socio-technical System?}

As a conclusion to this section, we will consider Cannon's six propositions [14], listed in Section 2.2, and see whether we can explain how they are realized in a socio-technical system in terms of FEM. The first step to relating Cannon's propositions to our work is to establish who the agencies/agents referred to in the propositions are. As we discussed in Section 3, each process node with assets attached to it represents a socio-technical system called BPWS (Business Process Work System). It is responsible for starting BPIs (Business Process Instances) of the given type, giving them assets required for their completion and overseeing their work (e.g. cancelling the running BPIs when necessary). The actions of a BPWS are steered by changes in the internal or external environment. For instance, an instance of an acquire process is started 
when the corresponding asset is depleted or there is an indication that it might be depleted soon. In the same way, an already started instance of the process can be stopped, as the need for increasing the size of the assets disappears. In the discussion below, we consider an agency/agent in Canon's propositions being equal to a BPWS.

The next concept from Canon's propositions that needs to be matched to FEM is "the factor." We believe that a factor could be considered as an action(s) of BPWS aimed at creating or stopping BPIs of the corresponding type.

Having established the mapping between the concepts from Cannon's propositions and FEM, we can now try to explain how Cannon's propositions manifest themselves in a socio-technical system from the FEM point of view. When giving an explanation, we also give a name to each proposition that expresses it in a concise form.

1. Acting agencies. As was discussed in Section 3, BPWSs are either acting, by having running BPIs, or being ready to act when a perturbation in an external or internal environment is detected by starting new BPIs.

2. Resistance to change. A tendency to change, like depletion of a certain asset, automatically invokes a factor that resists this change, such as an acquire process initiating an instance to fill the depleted asset with new element(s). See also explanation of Proposition 6 below, which is connected to this one.

3. Unidirectedness of actions. A BPI started by a BPWS has a goal which cannot be changed arbitrarily. For instance, a BPI of an acquire process cannot do anything else than try to get a new element of a corresponding asset. It cannot, for instance, retire an element from the same asset.

4. Cooperation of antagonistic agents. A retire and acquire BPWS managing the same asset work in opposite directions. However, they need to cooperate in order not to throw the system off balance. A retire BPWS has to provide information in advance that some elements of the asset will be soon retired in order to the acquire BPWS to start instances of the process in advance instead of waiting until the asset is actually depleted. For more on communication between the BPWSs that have at least one common asset see in [19].

5. Complex coordination of action. This principle expands principle 1 ; it is needed in order to adapt the system to more complex changes in the internal and external environment, such as appearing of new technology, or changing the educational level of the pool of people available for employment. This type of changes has already been discussed in Section 4.1. If the nature of an external pool from which an acquire process gets element to be bonded in the system changes, then other processes related to the corresponding assets need to be changed. This again requires communication between BPWSs related to the same asset as described in [19].

6. Opposing actions. In terms of FEM, there is a need for a mechanism that prevents abnormal behavior of a BPWS, such as an acquire process uncontrollably adding new elements to the corresponding asset (e.g. hiring new employees) or a retire process uncontrollably removing them from the pool (e.g. firing employees). For such a situation, just balancing an abnormal behavior of acquiring process by increasing the speed of the retiring process will not work as it will make the system unstable.

A standard way of dealing with such situations is by having a negative feedback loop that includes several, not necessarily antagonistic agents (BPWS). In terms of business process management, the correction can be done by tuning the rules that govern the process instances execution. This kind of rules are introduced via operational manuals, process descriptions, process maps, policies and other regulating documents. In FEM, such documents are represented as assets that are connected to the process, they govern, by arrows labeled EXT, where EXT stays for Executable Template, see Figure 2. Adapting EXT assets of each process when deviations are discovered can prevent the BPWSs unbalancing the system. 
In terms of FEM, such a solution can be represented as Figure 5. In the figure, the Process's behavior is regulated by Process manuals that serve as an asset of type EXT for the Process. The manuals are maintained by another process called Process improvement, and this process uses the traces of the Process's behavior as one of the bases for process improvement. The traces themselves are produced by the process instances started by the Process's BPWS, which is represented by the arrow of type Acquire from the Process to the asset Documented traces of behavior.

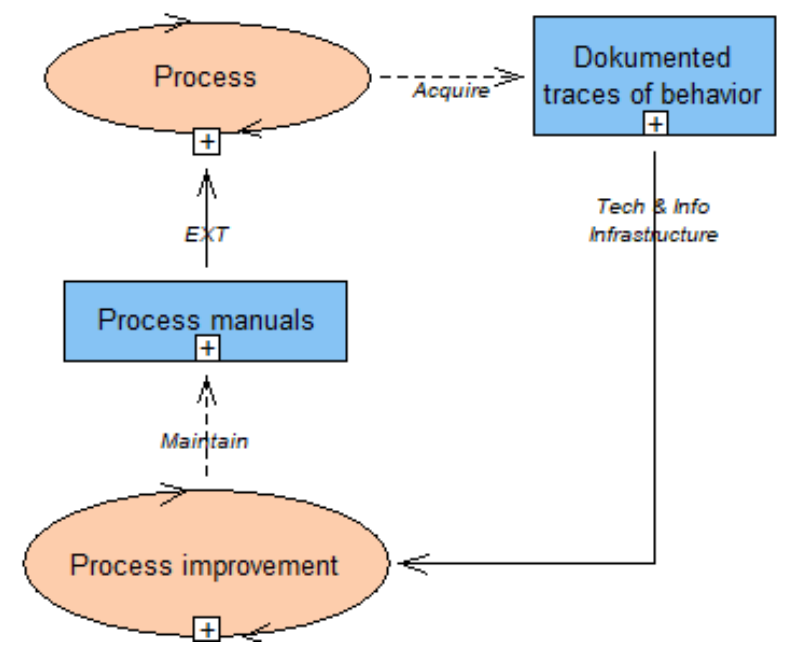

Figure 5. Negative feedback loop to restrain abnormal process behavior

\section{Discussion}

This article is devoted to the issues of autopoiesis and homeostasis in socio-technical systems. The first issue is about how a system constantly reproduces itself, while the second one is about how the system adapts itself to the perturbations in its internal and external environments while remaining itself. There is a large body of literature that deals with reproduction and adaptation. Why do we need more, what is the difference, and why the suggested approach might be useful?

\subsection{What is the Difference?}

As has been mentioned in the introduction, we use the POSWID principle when considering autopoiesis and homeostasis in organizational systems. More specifically, we focus on what an organization does, which, at a large extent, amounts to starting instances of various business processes to replenish the organizational assets. As the result, our work differs from the main body of autopoietic literature in two aspects:

(1) There is a tendency to exclude material parts of the system from consideration and concentrate on immaterial parts such as communication acts and decisions, as suggested by Luhmann [3], or knowledge, values, and beliefs. Our approach is focused on the reproduction of the (extended) body of the system, e.g. people being able to participate in processes, technology that helps to run the processes, customers who buy products and services, etc. This focus does not mean that we totally exclude and ignore the immaterial things. Communication and decision-making happen in the processes and need to be supported by technical tools, manuals and other types of documents as well as training of people to use the tools and follow the instructions. Knowledge is spread through EXT-types of assets, embedded in technical tools and, primarily, exists in the heads of people working in the process (tacit knowledge). People need to acquire specific knowledge before and during their participation in the process.

The focus on reproducing the "body" results in our view on the autopoietic system's closeness being somewhat different from those of others. We do not consider the system totally 
closed, or only consuming energy from the environment. For reproducing its body, the sociotechnical system needs to obtain and incorporate in its "body" quite complex elements, such as people, or technology. Despite this "openness", the system is still considered as operationally closed, as only the system itself can create and change its structure. The operational closure, as such, does not prevent the system from being destroyed (losing its identity) from the inside, or the outside. It only states that it cannot be forcefully changed from the outside in order to be better adjusted to the changes in its environment. Only the system itself could do it.

(2) Most of the literature discusses reproduction and adaptation in the abstract, often philosophical level. We focus on depicting how the reproduction and adaptation actually happen in a concrete enterprise or organization using a model that explains its operations. We want to show which processes are responsible for reproduction of which elements of the structure, and where from come elements of the environment that are needed to reproduce the body of a socio-technical system. We have shown that the answer to the questions above can be given by building a model that depicts the operational activities of the company. In this work, we have used the FEM technique for building a model, and the technique was extended to better suit the task at hand. This technique can also show how adaptation happens as a reaction on perturbations in the environment, especially those that concern external pools from which elements for reproduction are taken. The model can also explain how more radical changes happen when a system recouples itself to a different set of external pools while maintaining the major part of its coupling at each step. The latter can be considered as maintaining identity while changing.

\subsection{A Different View on Business Processes}

The very notion of business processes was put forward in the early 1990s with the seminal papers of the Business Process Reengineering movement [27], [28]. The reasoning back then was that of a set of activities that take some inputs and convert them into valuable output for a customer. Since then, however, the most common usage of business processes was as a means of optimizing the usage of organizational resources. This is done by optimizing the flow of activities, automating their transitions, balancing the usage of people and machines, improving communication and collaboration inside the frame of a process instance (removing the siloes), etc. In this article, business processes are considered as a means for reproducing the organizational body, detecting changes in the organizational environment, and adapting to these changes. We consider another view on business processes as the very agencies that ensure autopoiesis and homeostasis. This is a more strategic view of business processes as the mechanisms that insure the survival of the organization. It thus requires equal, if not more attention from both researchers and practitioners. Focusing only on optimization of individual processes may result in degradation of total organizational performance due to the loss of connection between different processes.

To the best of our knowledge, there is not much literature that highlights the role of business processes for the maintenance of identity, with the notable exceptions of [29], [30], [31], [32].

\subsection{Practical Implications}

A potential impact of this work on practice requires a separate investigation, but some areas of practical application can be identified even at this early stage. In this section, we consider one example of such areas.

One of the important results of this article is highlighting the needs of reproducing the body of the organization while keeping the alignment between the assets used in the same business 
process $^{\dagger}$. Consider, for instance, an asset of type Workforce engaged in a given business process. If the turnover of this asset is low, i.e. people leave and are hired rarely, then the process may rely on tacit knowledge in the heads of the people engaged in the process. This knowledge can be effectively transferred to the new members on the tacit level as well, e.g. using apprenticeship. In terms of FEM, it means that there is a low level of needs to have explicitly written instructions, handbooks, manuals, i.e. assets of type EXT. However, if the turnover of staff is high, there is a greater need for strict regulating mechanisms for the process in the form of EXT assets or built in software or hardware systems (assets of the Infrastructure type). The same situation emerges in an expansion phase, when a considerable number of new employees are expected to join the company. Creating EXT assets for this phase becomes an emergency.

When considering an expansion, or functioning with a high turnover of workforce, there is a need to investigate to what degree the current process can rely on explicit instructions vs. tacit knowledge. For instance, developing a software in an agile way requires a stable team of experienced developers that can promptly adjust their way of working to the nature of the software to be developed. Trying to substitute such a team with a strictly regulated process may completely remove the essence of the agile principles from software development, which, unfortunately, happens quite often.

As we already mentioned in the introduction, the practical usability of this work depends on the background of the user and his/her position in the organizational world. For instance, we are fully aware that the issues discussed above are well-known to experienced managers and expert management consultants. However, they might be invisible to less experienced people, e.g. new entrepreneurs. In addition, even experienced people can have some usage of our work, as it may give some theoretical underpinning to their practical knowledge. This might make it easier for them to give an explanation when promoting their suggestions to less experienced decision makers. Using FEM to visualize a specific area of consideration might also help for this end.

\section{Concluding Remarks}

In the introduction, we have posed three questions regarding autopoiesis and homeostasis in socio-technical systems:

1. What is reproduced (body)?

2. How is it done (network of processes)?

3. How the autopoietic activities help with maintaining homeostasis, i.e. adapting the system to the changes inside and outside the system while maintaining its identity.

Based on FEM, the answer to the first question is that the system (i.e. an enterprise or any other type of organizations) reproduces its assets, which include people (e.g. workforce), technology (infrastructure), methods (EXT), and even a more intangible assets, like reputation. We can answer the second question also based on FEM, namely, the reproduction is done by processes of the type acquire, some of them taking elements to be bonded in the system from the external environment.

As follows from the discussion in Section 4, there are two ways for an organization to adapt to perturbations in its environment. One is changes in the pools from which the acquire processes take material for reproduction. Such changes may require adjustment of processes that rely on assets managed by these acquire processes. The second type of adaptation is when the system changes its structural coupling with the external environment. This kind of change consists in (a) creating new processes that require different kind of assets, and, as a consequence, new acquire processes connected to new external pools; (b) dropping some processes and thus losing

\footnotetext{
$\dagger$ Note that alignment between components of a socio-technical system is one of the main topics in the socio-technical systems research. For instance, both classical socio-technical matrix from [9], and extended socio-technical matrix from [33], could be considered as a tool of analyzing and achieving alignment between the components of the socio-technical system. Our current work adds an additional dimension - alignment should be maintained while the body is constantly reproduced.
} 
connections to the external pools that provided material to the assets solely used in these processes. As we show in Section 4, using FEM can help in understanding both kinds of changes. The processes of deciding on and implementing the changes remain outside FEM, nevertheless FEM can help in detecting the need for a change, generating hypothesis [20], and planning and completing the change.

As far as using enterprise modeling as a tool for understanding autopoiesis and homeostasis in organizational (socio-technical) systems is concerned, we consider the results as positive. Without this tool, it would be difficult to give clear answers on the three questions above. Using FEM has helped us to visualize the ideas that were discussed in the article. The question whether some other modeling technique could be used for explaining the notions of autopoiesis and homeostasis remains open. Changing the modeling technique may result in a different view on what is being reproduced and how the system adapts.

This research is a concatenation of two initiatives, FEM and autopoiesis (Bider and Perjons [19]), and organizational modeling with homeostasis (Regev [16]). This research is also part of a broader undertaking connected to FEM. Initially, FEM has been developed as a means for finding all or the majority of the processes that exist in an organization. The result of this research produced more than a solution to the original problem, as FEM includes not only relations between the processes, but produces a map of assets usage and management in the organization. We therefore continued our work on FEM looking for other problems/challenges that can be solved using FEM and enhancing FEM when necessary. One example of a specific application of FEM is using it for business model innovation, see, for instance [17]. While our other research projects concerned practical application of FEM, this work is about using it for investigating theoretical concepts, i.e. autopoiesis and homeostasis. The investigation resulted in extending FEM with new concepts described in Section 5.3, which constitutes an additional contribution - side effect - of the current work. The extensions allow presenting external context in FEM, which opens new ways of using FEM in practice. In particular, we are currently working on using FEM for discovering structural couplings of an organization.

\section{References}

[1] F. J. Varela, H. R. Maturana, and R. Uribe, "Autopoiesis: The organization of living systems, its characterization and a model," BioSystems, vol. 5, no. 4, pp.187-196, 1974. Available: https://doi.org/10.1016/0303-2647(74)90031-8

[2] H. R. Maturana and F. J. Varela, Autopoiesis and Congnition: The Realization of the Living, Reidel, Dordrecht, Holland: Reidel, 1980.

[3] N. Luhmann, "The autopoiesis of social systems," Sociocybernetic paradoxes. London: Sage, 1986.

[4] R. Magalhaes and R. Sanchez, "Autopoiesis in organizational theory and practice," Advanced Series in Management, vol. 6, Bradford: Emerald Group, 2010. Available: https://doi.org/10.1108/S18776361(2009)0000006021

[5] S. Blaschke, "Chapter 12 The Autopoiesis of Organizational Knowledge, Learning, and Memory", Advanced Series in Management, vol. 6, Bradford: Emerald Group, pp. 215-231, 2010. Available: https://doi.org/10.1108/S1877-6361(2009)0000006013

[6] M. Zeleny, "On social nature of autopoietic system," Evolution, Order and Complexity. Taylor \& Francis Group, pp. 122-145, 1996.

[7] H. Cadenas and M. Arnold, "The autopoiesis of social systems and its criticisms," Constructivist Foundations, vol. 10, no. 2, pp. 169-176, 2015.

[8] N. Virgo, "The necessity of extended autopoiesis," Adaptive Behaviour, pp. 1-4, 2019. Available: https://doi.org/10.1177/1059712319841557

[9] R. P. Bostrom and J. S. Heinen, "MIS problems and failures: A socio-technical perspective," MIS Quarterly, vol. 1 , no. 3, pp. 17-32, 1977. Available: https://doi.org/10.2307/248710

[10]P. Hoverstadt, "Defining identity by structural coupling in VSM practice," UK Systems Society. Oxford, 2010.

[11] S. Beer, The Heart of Enterprise. Wiley, 1979. 
[12]I. Bider, E. Perjons, M. Elias, and P. Johannesson, "A fractal enterprise model and its application for business development," Software \& Systems Modeling, vol. 16, pp. 663-689, $2016 . \quad$ Available: https://doi.org/10.1007/s10270-016-0554-9

[13]I. Bider, G. Regev, and E. Perjons, "Linking autopoiesis to homeostasis in socio-technical systems," STPIS'19: Socio-Technical Perspective in IS Development 2019. CEUR, pp. 160-70, 2019.

[14] W. B. Cannon, “Organization for physiological homeostasis,” Physiological Reviews, vol. IX, no. 3, pp. 399431, 1929. Available: https://doi.org/10.1152/physrev.1929.9.3.399

[15] W. B. Cannon, The Wisdom of the Body. New York: Norton \& Company, 1939.

[16] G. Regev, O. Hayard, and A. Wegmann, "What We Can Learn About Business Modeling From Homeostasis," Business Modeling and Software Design. BMSD 2012. LNBIP, Springer, vol. 142, pp. 1-15, 2013. Available: https://doi.org/10.1007/978-3-642-37478-4_1

[17]I. Bider and E. Perjons, "Defining Transformational Patterns for Business Model Innovation," Perspectives in Business Informatics Research: 17th International Conference, BIR 2018, Stockholm, Sweden, LNBIP, Springer, vol. 330, pp. 81-95, 2018. Available: https://doi.org/10.1007/978-3-319-99951-7_6

[18]I. Bider and A. Chalak, "Evaluating Usefulness of a Fractal Enterprise Model Experience Report," Enterprise, Business-Process and Information Systems Modeling. BPMDS 2019, EMMSAD 2019, LNBIP. Springer, vol. 352, pp. 359-373, 2019. Available: https://doi.org/10.1007/978-3-030-20618-5_24

[19] I. Bider and E. Perjons, "Using Fractal Enterprise Model to Assist Complexity Management," BIR Workshops 2018, CEUR, pp. 233-238, 2018.

[20] S. Alter, The Work system method: Connecting people, processes, and IT for business results. Work System Press, 2006.

[21] H. W. Lawson, A journey through the systems landscape. College Publications, 2010.

[22] S. Albert and D. A. Whetten, "Organizational identity," Research in Organizational Behavior. Oxford: Elsevier, pp. 263-295, 1985.

[23] D. A. Whetten, "Albert and Whetten Revisited. Strengthening the Concept of Organizational Identity," Journal of Management Inquiry, vol. 15, no. 3, pp. 219-234, $2006 . \quad$ Available: https://doi.org/10.1177/1056492606291200

[24] J. Y. Lettvin, H. R. Maturana, W. S. McCulloch, and W. H. Pitts, "What the Frog's Eye Tells the Frog's Brain," Proceedings of the Institute of Radio Engineers, vol. 47, pp. 1940-1951, 1959. Available: https://doi.org/10.1109/JRPROC.1959.287207

[25] H. Maturana, "Autopoiesis, structural coupling and cognition,” Cybernetics \& Human Knowing, vol. 9, no. 34, pp. 5-34, 2002.

[26]I. Bider and E. Perjons, "Using structural coupling approach for defining and maintaining identity of an educational institution. Experience report," STPIS 2018. 4th International Workshop on Socio-Technical Perspective in IS development (STPIS'18), CEUR, pp. 24-39, 2018.

[27] M. Hammer, “Reengineering work: Don't automate, obliterate,” Harvard Business Review, July-August, 1990.

[28] T. H. Davenport and J. E. Short, "The new industrial engineering: information technology and business process redesign," MIT Sloan Management Review, July, 1990.

[29] G. Regev, I. F. Alexander, and A. Wegmann, "Modelling the regulative role of business processes with use and misuse cases," Business Process Management, vol. 11, no. 6, pp.695-708, 2005. Available: https://doi.org/10.1108/14637150510630864

[30] G. Regev, I. Bider, and A. Wegmann, "Defining Business Process Flexibility with the help of Invariants," Software Process: Improvement and Practice (SPIP), vol. 12, no. 1, pp. 65-79, 2007. Available: https://doi.org/10.1002/spip.301

[31] G. Regev and A. Wegmann, "A regulation-based view on business process and supporting system flexibility," Proceedings of the CAiSE'05 Workshops, pp. 91-98, 2005.

[32] M. M. Narasipuram, G. Regev, K. Kumar and A. Wegmann, "Business process flexibility through the exploration of stimuli," International Journal of Business Process Integration and Management (IJBPIM), vol. 3, no. 1, pp. 36-46. Available: https://doi.org/10.1504/IJBPIM.2008.019346

[33] I. Bider, and V. Klyukina, "Using a Socio-Technical Systems Approach for a Sales Process Improvement," IEEE 22nd International Enterprise Distributed Object Computing Workshop (EDOCW), pp. 48-58, 2018. Available: https://doi.org/10.1109/EDOCW.2018.00019 